\title{
Spin-Hall conductivity and electric polarization in metallic thin films
}

\author{
Xuhui Wang, ${ }^{1}$ Jiang Xiao (萧江), ${ }^{2,3,}{ }^{*}$ Aurelien Manchon, ${ }^{1}$ and Sadamichi Maekawa ${ }^{4,5}$ \\ ${ }^{1}$ King Abdullah University of Science and Technology (KAUST), Physical Science and Engineering Division, \\ Thuwal 23955-6900, Saudi Arabia \\ ${ }^{2}$ Department of Physics and State Key Laboratory of Surface Physics, Fudan University, Shanghai 200433, China \\ ${ }^{3}$ Center for Spintronic Devices and Applications, Fudan University, Shanghai 200433, China \\ ${ }^{4}$ Advanced Science Research Center, Japan Atomic Energy Agency, Tokai 319-1195, Japan \\ ${ }^{5}$ CREST, Japan Science and Technology Agency, Tokyo 102-0075, Japan
}

(Received 23 October 2012; revised manuscript received 4 February 2013; published 21 February 2013)

\begin{abstract}
We predict theoretically that when a normal metallic thin film (without bulk spin-orbit coupling, such as $\mathrm{Cu}$ or $\mathrm{Al}$ ) is sandwiched by two insulators, two prominent effects arise due to the interfacial spin-orbit coupling: a giant spin-Hall conductivity due to the surface scattering and a transverse electric polarization due to the spin-dependent phase shift in the spinor wave functions.
\end{abstract}

DOI: 10.1103/PhysRevB.87.081407

PACS number(s): 72.25.Ba, 75.70.Tj, 75.75.-c

Spin-orbit interaction, transferring angular momentum between electronic spins and orbital motion, has extended the boundary of the field of spintronics towards a full-electric manipulation of spins without using magnets. By coupling the charge and spin currents, spin-orbit interaction has left its signature in bulk metals ${ }^{1-3}$ and semiconductors ${ }^{4-8}$ by the so-called spin-Hall effect, ${ }^{9}$ which catches much attention in academia and industry due to its interesting physics and potential applications.

In noble metals such as Pt and $\mathrm{Au}$, a strong bulk spin-orbit coupling drives the (inverse) spin-Hall effect, which has been thoroughly studied in numerous settings, providing an alternative to generate or detect spin current. ${ }^{10-13}$ Theoretical investigations, $a b$ initio calculations in particular, have strengthened our understanding of the role played by bulk impurities ${ }^{14,15}$ and doped surfaces. ${ }^{16}$ With a series of efforts to dope $\mathrm{Cu}$ using spin-orbit scatterers (see Ref. 17 and references therein), a large spin-Hall angle has been reported in $\mathrm{Cu}$ thin film with bismuth impurities recently. ${ }^{18}$ In this Rapid Communication, we propose an alternative method to realize the spin-Hall effect in $\mathrm{Cu}$ without doping but by sandwiching the $\mathrm{Cu}$ thin film with two dissimilar insulators such as oxides or even vacua. The inversion symmetry breaking across the interfaces provides interfacial spin-orbit couplings (ISOCs), thus allowing metals such as $\mathrm{Al}$ or $\mathrm{Cu}$ to accommodate a spin-Hall conductivity that may be even larger than that caused by a bulk spin-orbit interaction in noble metals. Meanwhile, we demonstrate that the ISOC also induces an in-plane wave-vector-dependent spatial separation of wave functions between opposite spin types along the confinement direction. Combined with the spin imbalance (between the majority and minority bands) due to the structural asymmetry, such a spatial separation induces a transverse electric polarization that is quadratic in the in-plane electric field.

A schematic picture of the setup is shown in Fig. 1. The normal metal film has a constant thickness $d$ along the transverse direction $(z)$, while two interfaces are in the $x y$ plane located at $z_{ \pm}= \pm d / 2$. The confinement of the insulators is described by finite potential steps,

$$
V_{C}=V_{+} \theta\left(z-z_{+}\right)+V_{-} \theta\left(z_{-}-z\right),
$$

where $V_{ \pm}$is the height of the potential barrier at $z_{ \pm}= \pm d / 2$ and $\theta(z)$ is the Heaviside step function. We are interested in the surface scattering by the Rashba-type spin-orbit interaction ${ }^{20}$ generated at interfaces between a normal metal film and insulating materials, such as metal oxides. The potential barriers Eq. (1) generate two electric fields $\boldsymbol{E}_{C}=-\nabla V_{C}$ that are localized at the interfaces and aligned oppositely to each other along the $z$ direction, giving rise to a Rashba-type ISOC,

$H_{R}=\frac{\lambda_{-} V_{-} \delta\left(z-z_{-}\right)-\lambda_{+} V_{+} \delta\left(z-z_{+}\right)}{\hbar}\left(\hat{\sigma}_{x} \hat{p}_{y}-\hat{\sigma}_{y} \hat{p}_{x}\right)$,

where $\lambda_{ \pm}$is the spin-orbit coupling parameter for the corresponding interface, $\hat{\sigma}$ is the Pauli matrix, and $\hat{\boldsymbol{p}}$ is the canonical momentum.

Band structure and wave function. The full Hamiltonian for this system is $H=p^{2} /(2 m)+V_{C}+H_{R}$. We first treat the ISOC $H_{R}$ with degenerate perturbation. Using the wave functions of a finite potential well and a standard degenerate perturbation technique, ${ }^{21}$ assuming the energy splitting due to the ISOC is much smaller than the interchannel energy spacing, we obtain the energy eigenvalue and wave function for an eigenspinor labeled by spin polarization $s= \pm$, an in-plane wave vector $\boldsymbol{k}$, and a transverse channel index $n$,

$$
\begin{aligned}
& E_{n \boldsymbol{k} s}=\frac{\hbar^{2} k^{2}}{2 m}+E_{0} n^{2}+s \frac{2}{d_{e}} k\left|\lambda_{+}-\lambda_{-}\right| E_{0} n^{2}, \\
& \psi_{n \boldsymbol{k} s}=\frac{1}{\sqrt{A d_{e}}} e^{i \boldsymbol{k} \cdot \boldsymbol{r}} \sin \left(\frac{n \pi}{d_{e}} z+\delta\right)\left(\begin{array}{c}
1 \\
-i s e^{i \theta}
\end{array}\right),
\end{aligned}
$$

where $\tan \theta=k_{y} / k_{x}, E_{0} \equiv \hbar^{2} \pi^{2} /\left(2 m d_{e}^{2}\right)$, and $A$ is the area of the metal film. A schematic view of the band energy and the spatial part of the wave function is shown in Fig. 2. The spatial part of the wave function $\psi_{n k s}$ in Eq. (3b) is the same for majority $(s=-)$ and minority $(s=+)$ spins in the first-order perturbation calculation. The phase shift for both spins is given by $\delta=n \pi / 2+\left(d_{+}-d_{-}\right) n \pi /(2 d)$ with $d_{ \pm}=\hbar / \sqrt{2 m V_{ \pm}}$the approximate penetration depth into the barrier. By assuming high confinement barriers $\left(V_{ \pm} \gg E_{F}\right.$, given $E_{F}$ the Fermi energy), the effective thickness $d_{e}=d+d_{+}+d_{-}$. Therefore, a finite potential well of thickness $d$ can be viewed equivalently as an infinite well with thickness $d_{e}$. We shall point out that, although the eigenenergies for majority and minority are 


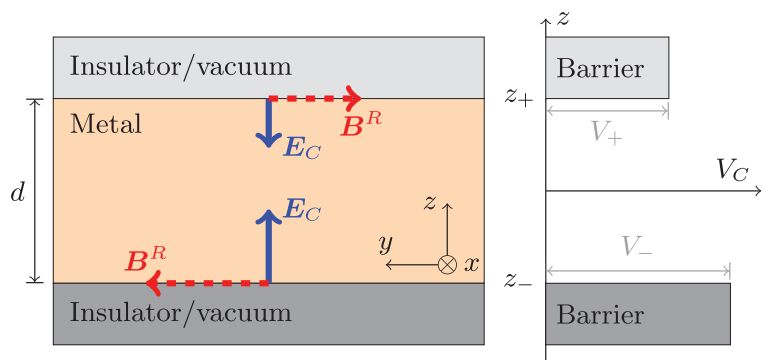

FIG. 1. (Color online) Left: A metallic film of thickness $d$ is sandwiched by two dissimilar insulating materials, such as oxides or vacua. The electric fields (solid blue arrows) at the interfaces point inward $( \pm z)$, and the resulting Rashba magnetic fields (dashed red arrows) point sideward $( \pm y)$ for an electron moving along the $x$ direction. Right: The confining potential $V_{C}$ in Eq. (1) along the confinement direction (i.e., $z$ axis).

different, such a difference does not lead to any magnetism because of the exact cancellation among spins at different $\boldsymbol{k}$ directions.

Spin-Hall conductivity. When $\lambda_{+} \neq \lambda_{-}$, for each conducting channel (or transverse mode), the electron is characterized by a free Hamiltonian $H_{0}^{(n)}=\boldsymbol{p}^{2} /(2 m)+E_{0} n^{2}$ augmented by an effective momentum-dependent Rashba magnetic field $\boldsymbol{B}_{n}^{\mathrm{R}}(\boldsymbol{p})=\left(2 \lambda_{n}^{\text {eff }} / \hbar\right)(\hat{z} \times \boldsymbol{p})$ with a channel-dependent coupling constant $\lambda_{n}^{\text {eff }}=2\left(\lambda_{+}-\lambda_{-}\right) E_{0} n^{2} / d_{e}$. For an electron in band $n$ carrying an in-plane momentum $\boldsymbol{p}$, assuming $\lambda_{+}>\lambda_{-}$, the majority (minority) spin points to the $\boldsymbol{B}_{n}^{\mathrm{R}}\left(-\boldsymbol{B}_{n}^{\mathrm{R}}\right)$ direction.

When an electric field is applied along the $\hat{x}$ axis, the Rashba field $\boldsymbol{B}_{n}^{\mathrm{R}}(\boldsymbol{p}(t))$ becomes time dependent since $\dot{p}_{x}(t)=-e E_{x}{ }^{5}$ In the rotating frame that follows $\boldsymbol{B}_{n}^{\mathrm{R}}(\boldsymbol{p}(t))$, the time dependence is translated into a gauge field $\boldsymbol{B}_{n}^{\mathrm{G}}=-e \hbar p_{y} E_{x} / p^{2} \hat{z} \cdot{ }^{19}$ In the adiabatic limit ( $e E_{x} \ll \lambda_{n}^{\text {eff }} k_{F}^{2}$ ), the majority (minority) spins align (antialign) with the total field $\boldsymbol{B}_{n}^{\mathrm{T}}=\boldsymbol{B}_{n}^{\mathrm{R}}+\boldsymbol{B}_{n}^{\mathrm{G}}$, resulting in an out-of-plane $(\hat{z})$ spin component

$$
n_{z}^{s}(\boldsymbol{p}) \approx s \frac{B_{n}^{\mathrm{G}}}{B_{n}^{\mathrm{R}}}=-s \frac{e \hbar^{2} p_{y} E_{x}}{2 \lambda_{n}^{\text {eff }} p^{3}} .
$$
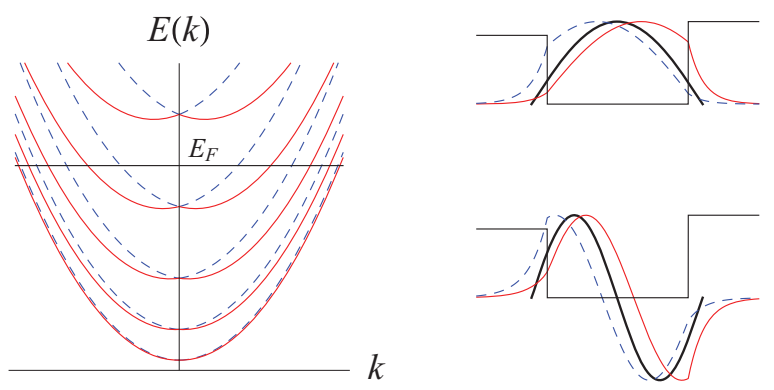

\section{$k$}

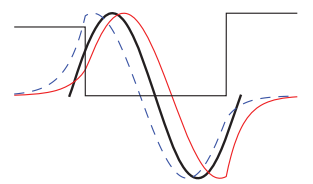

FIG. 2. (Color online) Left: Schematic view of the band structure in metallic thin film with interfacial Rashba spin-orbit coupling, majority (solid red) and minority (dashed blue). Right: Schematic picture of the spatial part of the wave function for the first two subbands; the stepwise curve is the confinement potential profile and the three different curves are the wave function from the lowest-order perturbation Eq. (3b) (solid black for both majority and minority) and the exact wave function from Eq. (10) (solid red for majority and dashed blue for minority) which shows the spatial separation of opposite spins. The discontinuity at the interfaces is due to the $\delta$-function-like potential in $H_{R}$.
Consequently, in a metal film of volume $\mathcal{V}=d A$, at the Fermi level, the spin current polarized in the $\hat{z}$ direction while flowing in the $\hat{y}$ direction is

$$
j_{y}^{z}=\frac{1}{\mathcal{V}} \sum_{n, \boldsymbol{p}, s} \frac{\hbar}{2} n_{z}^{s}(\boldsymbol{p}) \frac{p_{y}}{m}=-\frac{e n_{c}}{8 \pi d} E_{x} .
$$

Therefore, the spin-Hall conductivity caused by ISOC is

$$
\sigma^{\mathrm{SH}}=\frac{e}{8 \pi} \frac{n_{c}}{d} \approx \frac{e}{8 \pi^{2}} k_{F},
$$

where $n_{c}=\left\lfloor k_{F} d / \pi\right\rfloor$ is the total number of transverse channels. The symbol $\lfloor a\rfloor$ denotes the largest integer that is smaller than $a$. Note that Eq. (6) is obtained for $\lambda_{+}>\lambda_{-}$, the sign of $\sigma^{\mathrm{SH}}$ should change when $\lambda_{+}<\lambda_{-}$, and $\sigma^{\mathrm{SH}}=0$ for $\lambda_{+}=\lambda_{-}$.

To extend the semiclassical picture above, we employ the Kubo formula to calculate the dc spin-Hall conductivity (i.e., $\omega \rightarrow 0$ ) in the linear response regime: ${ }^{5}$ An electric current flowing along the $x$ direction gives rise to a spin current that is polarized to the $z$ axis while transporting along the $y$ direction,

$$
\begin{aligned}
\sigma^{\mathrm{SH}}= & \frac{e \hbar}{V} \sum_{\boldsymbol{k}} \sum_{n, n^{\prime}} \sum_{s \neq s^{\prime}}\left(f_{n^{\prime} \boldsymbol{k} s^{\prime}}-f_{n \boldsymbol{k} s}\right) \\
& \times \frac{\operatorname{Im}\left[\left\langle\psi_{n^{\prime} \boldsymbol{k} s^{\prime}}\left|\hat{j}_{y}^{z}\right| \psi_{n \boldsymbol{k} s}\right\rangle\left\langle\psi_{n \boldsymbol{k} s}\left|\hat{v}_{x}\right| \psi_{n^{\prime} \boldsymbol{k} s^{\prime}}\right\rangle\right]}{\left(E_{n \boldsymbol{k} s}-E_{n^{\prime} \boldsymbol{k} s^{\prime}}\right)\left(E_{n \boldsymbol{k} s}-E_{n^{\prime} \boldsymbol{k} s^{\prime}}-\hbar \omega-i \eta\right)},
\end{aligned}
$$

where $f_{n k s}$ is the electron spin occupation number. Using the Heisenberg equation, the in-plane velocity operator is given by

$$
\hat{v}_{x(y)}=\frac{\hbar}{i m} \nabla_{x(y)} \mp \frac{\lambda_{-} V_{-} \delta\left(z-z_{-}\right)-\lambda_{+} V_{+} \delta\left(z-z_{+}\right)}{\hbar} \hat{\sigma}_{y(x)},
$$

where the second term is the anomalous velocity due to the spin-orbit coupling that is localized at the interface. The definition of spin current polarized along the $z$ direction is $\hat{\boldsymbol{j}}^{z}=(\hbar / 4)\left\{\hat{\sigma}_{z}, \hat{\boldsymbol{v}}\right\}$. Using the eigensolutions in Eq. (3), we have

$$
\sigma^{\mathrm{SH}}=\frac{e}{8 \pi} \frac{\lambda_{+}-\lambda_{-}}{\left|\lambda_{+}-\lambda_{-}\right|} \frac{n_{c}}{d}= \pm \frac{e}{8 \pi} \frac{n_{c}}{d} \approx \pm \frac{e}{8 \pi^{2}} k_{F},
$$

where $\pm=\operatorname{sgn}\left(\lambda_{+}-\lambda_{-}\right)$and the last approximate value assumes a large number of transverse channels $n_{c} \gg 1$. For two perfectly identical interfaces $\lambda_{-}=\lambda_{+}$, the spin-Hall conductivity shall vanish. Equation (9) calculated from Kubo formula agrees with Eq. (6) derived semiclassically and comprises one of the main results of this communication.

To describe a spin-Hall system, one quantity often scrutinized in experiments is the spin-Hall angle defined as the ratio between $\sigma^{\mathrm{SH}}$ and the longitudinal conductivity $\sigma^{\mathrm{N}}$, characterizing the efficiency of converting charge (spin) current into spin (charge) current in a (inverse) spin-Hall system. For a coherent ballistic conductor presented in this communication, the dc longitudinal conductance (or resistance) measured in an experiment is dominated by scattering events at contacts. ${ }^{22}$ Therefore, we expect the spin-Hall angle to depend on the specific geometry and material selection of the contacts.

Transverse electric polarization. We now turn to an inviting effect, as caused by ISOC, that has not been discussed before to our knowledge. The ISOC in Eq. (2) not only gives rise to an energy splitting for opposite spins, but also separates the wave functions in real space; i.e., majority and minority 
spins are shifted towards opposite surfaces. This can be easily understood as the following: Assuming $\lambda_{+}>\lambda_{-}$, the potential barrier at surface $z_{+}\left(z_{-}\right)$is decreased (increased) by a $\delta$-function Rashba potential for majority spins; therefore majority spins tend to shift towards surface $z_{+}$. On the opposite, the minority spins shift toward surface $z_{-}$. Therefore, majority and minority spins are spatially separated along the transverse direction as shown schematically in Fig. 2. To quantify such an effect, the approximated wave function in Eq. (3b) is not enough, and we must seek an exact eigensolution to the full Hamiltonian $H$ of the following form (inside the metal film):

$$
\psi_{n \boldsymbol{k} s}=c_{n \boldsymbol{k} s} e^{i \boldsymbol{k} \cdot \boldsymbol{r}} \sin \left(q_{n \boldsymbol{k} s} z+\delta_{n \boldsymbol{k} s}\right)\left(\begin{array}{c}
1 \\
-i s e^{i \theta}
\end{array}\right),
$$

with normalization factor $c_{n k s}$ and the expression outside the metal is written similarly but with evanescent wave function in the $z$ direction. In the limit of $\lambda_{ \pm} k_{F}^{2} \ll 1$ and $E_{F} \ll V_{ \pm}$,

$$
\begin{aligned}
& q_{n k s} \approx \frac{n \pi}{d}+\frac{n \pi}{d^{2}}\left[\operatorname{sk}\left(\lambda_{+}-\lambda_{-}\right)-\left(d_{+}+d_{-}\right)\right], \\
& \delta_{n k s} \approx \frac{n \pi}{2}-\frac{n \pi}{2 d}\left[\operatorname{sk}\left(\lambda_{+}+\lambda_{-}\right)-\left(d_{+}-d_{-}\right)\right] .
\end{aligned}
$$

The spin-dependent transverse wave vector $q_{n \boldsymbol{k}}$ gives the energy splitting as in Eq. (3a) for the majority and minority spins with $E_{n k s}=\left(\hbar^{2} / 2 m\right)\left(q_{n k s}^{2}+k^{2}\right)$. The spin-dependent phase shift $\delta_{n k s}$ means that the wave function for majority (minority) spins with $s=-(+)$ is shifted toward negative (positive) $z$ direction. The transverse shift is spin and $k$ dependent, and can be quantified by the center of probability in the $z$ direction:

$$
z_{n s}(\boldsymbol{k})=\int z\left|\psi_{\boldsymbol{k} n s}(z)\right|^{2} d z \approx s k \frac{\lambda_{+}+\lambda_{-}}{2} .
$$

The last approximation omits a constant $\left(d_{+}-d_{-}\right) / 2$ that does not contribute to the nonequilibrium properties discussed below. For highly asymmetric interfaces (i.e., $\lambda_{+} \gg \lambda_{-}$), the transverse shift $\left|z_{n+}(\boldsymbol{k})-z_{n-}(\boldsymbol{k})\right| \propto \eta(k) d$, where $\eta(k)$ is the ratio between the interfacial Rashba energy splitting [third term in Eq. (3a)] and transverse channel energy spacing [second term in Eq. (3a)]. Therefore, by a realization of large $\eta$, the majority and minority spin channels can be spatially separated, and the spin-flip scattering is thus suppressed.

We now discuss the effect of the transverse shift on the electric polarization. What interests us the most is the nonequilibrium response of the electric polarization as a direct consequence of the ISOC. Because of the $k$ dependence, the transverse shift $z_{n s}(\boldsymbol{k})$ depends on the application of a current; therefore a nonequilibrium transverse electric polarization response to the in-plane electric field $\boldsymbol{E}$ can be calculated as $P_{z}(\boldsymbol{E})=\frac{e}{\mathcal{V}} \sum_{n \boldsymbol{k} s}\left[z_{n s}(\boldsymbol{k}+\delta \boldsymbol{k})-z_{n s}(\boldsymbol{k})\right]$ with $\delta \boldsymbol{k}=e \tau \boldsymbol{E} / \hbar$ :

$$
\begin{aligned}
P_{z}(\boldsymbol{E}) & =-\frac{e k_{F}^{3}}{3 d}\left(\frac{e \tau}{h}\right)^{2}\left(\lambda_{+}^{2}-\lambda_{-}^{2}\right) \boldsymbol{E} \cdot \boldsymbol{E} \\
& =-\frac{m^{2} e^{3}}{12 \pi^{2} \hbar^{4}}\left(\lambda_{+}^{2}-\lambda_{-}^{2}\right) \frac{k_{F}}{d} V^{2} .
\end{aligned}
$$

The second expression is for a coherent conductor with an electron dwelling time $\tau=l / v_{F}$ and $V=E l$. The quadratic dependence of $\boldsymbol{E}$ or $V$ is a result of symmetry in the in-plane dimensions.

In an analogy to Hall effects, the electric polarization Eq. (13) shall give rise to, across the confinement direction, a voltage signal that is quadratic in the (in-plane) applied voltage. The electric polarization Eq. (13) is a combination of two facts: (1) the spatial separation between majority and minority spins, which is proportional to $\lambda_{+}+\lambda_{-}$, and (2) the spin imbalance between the majority and minority spins, which is proportional to $\lambda_{+}-\lambda_{-}$. Equation (13) comprises the other main result of this communication.

We provide an estimate on the order of magnitude of the polarization density for the most asymmetric interfaces (e.g., $\lambda_{+} \neq 0$ and $\lambda_{-}=0$ ). The existing value of Rashba parameter in literature ranges from $\alpha_{R}=0.16 \mathrm{eV} \AA^{23}$ to $\alpha_{R}=2.5 \mathrm{eV} \AA,{ }^{24}$ depending on the materials. Here, we take $\alpha_{R}=0.16 \mathrm{eV} \AA$, corresponding to a Rashba energy splitting $\Delta_{R}=0.22 \mathrm{eV}$, for $\mathrm{Cu}$ with $k_{F}=1.36 \AA^{-1}$. We convert such an energy scale into $\lambda$ by $\Delta_{R} \approx 4 k_{F} \lambda E_{0} / d$. For a thin film of a thickness $d=10 \mathrm{~nm}$ and under an applied voltage, for example $V=100 \mathrm{nV}$, the polarization density $P_{z} \approx 2.1 \times 10^{-14} \mathrm{C} \mathrm{m}^{-2}$. Such an electric polarization is measurable (in a circuit enclosing two interfaces) under an ac in-plane electric field (or current): With a frequency $f=1 \mathrm{GHz}$, the magnitude of the induced current density in the transverse direction is $j_{z} \sim d P_{z} / d t \sim 10^{-5} \mathrm{~A} \mathrm{~m}^{-2}$. Meanwhile, the frequency of this induced current is doubled to $2 f$ because $P_{z}$ depends quadratically on $V$. Such a frequency doubling, as a consequence of the symmetry of the present system, is qualitatively different from Pershin and Di Ventra in Ref. 25, where the frequency doubling effect emerges from electron-electron interaction. In addition, we emphasize the electric polarization in our study arises from the phase shift of a single-electron wave function along the out-of-plane $z$ direction, while in Ref. 25, the electric polarization arises from a nonlinear effect due to many-body electron-electron interaction and an inhomogeneous charge density.

To the best of our knowledge, the transverse electric polarization induced by the ISOC predicted in this communication is a new effect that has not been discussed previously. This electric polarization manifests itself as a charge Hall effect and can be measured as a transverse voltage (or a current in a close circuit). Being qualitatively different from conventional charge Hall effects driven by Lorentz force in normal metals and anomalous Hall effect in ferromagnetic media, the electric polarization [Eq. (13)] is quadratic (instead of linear) in the longitudinal electric field and does not require external magnetic field or ferromagnetism.

Discussion and conclusion. The spin-Hall conductivity in Eq. (9) is derived for a ballistic sample where the bulk impurities are scarce. This is generally valid for thin films with thickness less than the mean free path and spin diffusion length. In an ultrathin film, surface roughness is a dominating scattering mechanism. ${ }^{26}$ In the present setting, the electric fields generated by the potential gradients are normal to interface while the presence of surface roughness effectively randomizes the field around an average direction that is still perpendicular to the average interface. Therefore, we can summarize the total effect of surface roughness into an effective spin-orbit coupling $\tilde{\lambda}_{i}$ that is smaller than $\lambda_{i}$. Since the leading order 
spin-Hall conductivity does not depend on $\lambda$ 's, we argue that the spin-Hall effect survives the roughness scattering. The seemingly abrupt jump in spin-Hall conductivity [i.e., Eq. (9)] at the point $\lambda_{+}=\lambda_{-}$is the manifestation of neglecting electron momentum relaxation in such a ballistic conductor. In a realistic setup, the sandwich-type conductor is always connected to reservoirs where the electrons are relaxed and a smoother change shall appear.

We need to point out that these results are, in many ways, different from the seminal works by Sinova et al. in a two-dimensional electron gas ${ }^{5}$ and Murakami et al. in a bulk semiconductor. ${ }^{4}$ First of all, in the present setup the spin-orbit coupling is neither intrinsic to the electrons (as in Ref. 5) nor arising from a particular band structure (as in Ref. 4), but due to the interface scattering. Second, our treatment to the spin-Hall conductivity is seemingly two-dimensional but the outcome highlights a bulk effect with a weak thickness $(d)$ dependence, as long as the structure can be treated coherently.

Third, and most importantly, because of the finite size in the $z$ direction, such a sandwich-type structure also accommodates a transverse electric polarization (being qualitatively different from the spin polarization studied by Edelstein $)^{27}$ through the ISOC, which is unique for the thin-film structure: The out- of-plane electric polarization along the $z$ direction does not exist in two-dimensional systems considered, for example, in Refs. 5,25, and 27.

In conclusion, we predict that in a coherent ballistic conductor that consists of an ultrathin normal metal (with negligible bulk spin-orbit coupling) film sandwiched by dissimilar insulators, the Rashba-type ISOC supports a spin-Hall effect featured by a large spin-Hall conductivity that is independent of the interfacial Rashba coupling. The ISOC also causes a spatial separation in the transverse wave functions of different spin bands. In response to an in-plane current or electric field, such a spatial separation gives rise to a transverse electric polarization that is quadratic in the in-plane field applied. The sandwich-type structure proposed in this communication has potential applications to replace noble metals (such as Pt) as a source and detector for spin currents.

We thank Gerrit E. W. Bauer, Xiaofeng Jin, and Mark Stiles for stimulating discussions. This work was supported by the National Natural Science Foundation of China (Grants No. 11004036 and No. 91121002) and the special funds for the Major State Basic Research Project of China (No. 2011CB925601).
*Corresponding author: xiaojiang@ fudan.edu.cn

${ }^{1}$ J. E. Hirsch, Phys. Rev. Lett. 83, 1834 (1999).

${ }^{2}$ S. Zhang, Phys. Rev. Lett. 85, 393 (2000).

${ }^{3}$ S. Takahashi and S. Maekawa, Phys. Rev. Lett. 88, 116601 (2002).

${ }^{4}$ S. Murakami, N. Nagaosa, and S.-C. Zhang, Science 301, 1348 (2003).

${ }^{5}$ J. Sinova, Dimitrie Culcer, Q. Niu, N. A. Sinitsyn, T. Jungwirth, and A. H. MacDonald, Phys. Rev. Lett. 92, 126603 (2004).

${ }^{6}$ Y. K. Kato, R. C. Myers, A. C. Gossard, and D. D. Awschalom, Science 306, 1910 (2004).

${ }^{7}$ G. E. W. Bauer, Science 306, 1898 (2004).

${ }^{8}$ J. Wunderlich, B. Kaestner, J. Sinova, and T. Jungwirth, Phys. Rev. Lett. 94, 047204 (2005).

${ }^{9}$ M. I. Dyakonov and V. I. Perel, Sov. Phys. JETP Lett. 13, 467 (1971); Phys. Lett. A 35, 459 (1971).

${ }^{10}$ S. O. Valenzuela and M. Tinkham, Nature (London) 442, 176 (2006).

${ }^{11}$ T. Kimura, Y. Otani, T. Sato, S. Takahashi, and S. Maekawa, Phys. Rev. Lett. 98, 156601 (2007).

${ }^{12}$ K. Ando, S. Takahashi, K. Harii, K. Sasage, J. Ieda, S. Maekawa, and E. Saitoh, Phys. Rev. Lett. 101, 036601 (2008).

${ }^{13}$ O. Mosendz, J. E. Pearson, F. Y. Fradin, G. E. W. Bauer, S. D. Bader, and A. Hoffmann, Phys. Rev. Lett. 104, 046601 (2010).

${ }^{14}$ M. Gradhand, D. V. Fedorov, P. Zahn, and I. Mertig, Phys. Rev. Lett. 104, 186403 (2010).
${ }^{15}$ S. Lowitzer, M. Gradhand, D. Kódderitzsch, D. V. Fedorov, I. Mertig, and H. Ebert, Phys. Rev. Lett. 106, 056601 (2011).

${ }^{16}$ B. Gu, I. Sugai, T. Ziman, G. Y. Guo, N. Nagaosa, T. Seki, K. Takanashi, and S. Maekawa, Phys. Rev. Lett. 105, 216401 (2010).

${ }^{17}$ Y. Niimi, M. Morota, D. H. Wei, C. Deranlot, M. Basletic, A. Hamzic, A. Fert, and Y. Otani, Phys. Rev. Lett. 106, 126601 (2011).

${ }^{18}$ Y. Niimi, Y. Kawanishi, D. H. Wei, C. Deranlot, H. X. Yang, M. Chshiev, T. Valet, A. Fert, and Y. Otani, Phys. Rev. Lett. 109, 156602 (2012).

${ }^{19}$ Y. Aharonov and A. Stern, Phys. Rev. Lett. 69, 3593 (1992).

${ }^{20} \mathrm{Yu}$. A. Bychkov and E. I. Rashba, JETP Lett. 39, 78 (1984).

${ }^{21}$ L. D. Landau and E. M. Lifshitz, Quantum Mechanics (NonRelativistic Theory) (Butterworth-Heinemann, Oxford, 1980).

${ }^{22}$ G. E. W. Bauer, Y. Tserkovnyak, D. Huertas-Hernando, and A. Brataas, Phys. Rev. B 67, 094421 (2003).

${ }^{23}$ A. G. Rybkin, A. M. Shikin, V. K. Adamchuk, D. Marchenko, C. Biswas, A. Varykhalov, and O. Rader, Phys. Rev. B 82, 233403 (2010).

${ }^{24}$ S. Mathias, A. Ruffing, F. Deicke, M. Wiesenmayer, I. Sakar, G. Bihlmayer, E. V. Chulkov, Yu. M. Koroteev, P. M. Echenique, M. Bauer, and M. Aeschlimann, Phys. Rev. Lett. 104, 066802 (2010).

${ }^{25}$ Y. V. Pershin and M. Di Ventra, Phys. Rev. B 79, 153307 (2009).

${ }^{26}$ Z. Tešanović, M. V. Jarić, and S. Maekawa, Phys. Rev. Lett. 57, 2760 (1986).

${ }^{27}$ V. M. Edelstein, Solid State Commun. 73, 233 (1990). 